Review

\title{
Potential Management of Circulating Tumor DNA as a Biomarker in Triple-Negative Breast Cancer
}

\author{
Mao Shang ${ }^{1,2}$, Chunxiao Chang ${ }^{3}$, Yanqing Pei ${ }^{4}$, Yin Guan ${ }^{5}$, Jin Chang ${ }^{6}$, HuiHui Li ${ }^{\circledR}$ \\ 1. School of Medicine and Life Sciences, University of Jinan Shandong Academy of Medical Sciences, Jinan, Shandong, China \\ 2. Department of Radiation Oncology, Shandong Cancer Hospital Affiliated to Shandong University, Shandong Academy of Medical Sciences, Jinan, \\ Shandong Province, China. 250117 \\ 3. Department of Medical Oncology, Shandong Cancer Hospital Affiliated to Shandong University, Shandong Academy of Medical Sciences, Jinan, Shandong \\ Province, China. 250117 \\ 4. Department of Quality Management Office, Shandong Cancer Hospital Affiliated to Shandong University, Shandong Academy of Medical Sciences, Jinan, \\ Shandong Province, China. 250117 \\ 5. Department of Medical Oncology, Beijing Chao-Yang Hospital, Beijing, China. \\ 6. Oncology department, Affiliated Hospital of Taishan Medical university.
}

$\square$ Corresponding author: Huihui Li, MD, PhD, Department of Medical Oncology, Shandong Cancer Hospital and Institute, Shandong Cancer Hospital affiliated to Shandong University, 440, Jinyan Road, Jinan, Shandong, China. Tel: 15553103209; Email address: 15553103209@163.com

(0) Ivyspring International Publisher. This is an open access article distributed under the terms of the Creative Commons Attribution (CC BY-NC) license (https://creativecommons.org/licenses/by-nc/4.0/). See http://ivyspring.com/terms for full terms and conditions.

Received: 2018.07.11; Accepted: 2018.09.11; Published: 2018.11.24

\begin{abstract}
As a specific subtype of breast cancer, Triple-negative breast cancer (TNBC) is associated with worse prognosis and higher tumor aggressiveness than HER2-amplified or hormone receptor positive breast cancers. Circulating tumor DNA (ctDNA), as a non-invasive "liquid biopsy", is an emerging original blood-based biomarker for early breast cancer diagnosis, monitoring treatment response, and determining prognosis. In TNBC patients, ctDNA has an inherent tendency to characterize tumor heterogeneity and metastasis-specific mutations providing a key alternative to tumor tissue profiling. Several studies have already demonstrated the potential of ctDNA in TNBC patients from early to advanced stages of the disease including diagnosis, therapy decisions and assessment of prognosis. This review provides a critical brief summary of the evidence that gives credence to the utility of ctDNA as a biomarker for its role into clinical management in TNBC.
\end{abstract}

Key words: triple-negative breast cancer, circulating tumor DNA, liquid biopsy

\section{Introduction}

Patients with triple-negative breast cancer (TNBC), which is negative for estrogen receptor (ER), progesterone receptor (PR), and human epidermal growth factor receptor 2 (HER2) expression, account for $15-20 \%$ of all breast cancer patients [1-3] and are generally considered to have poor prognosis $[4,5]$. Early diagnosis and appropriate treatment can improve the prognosis. Given that TNBC patients are not respondent to endocrine therapy and HER2 targeted therapy, chemotherapy is the mainstay treatment in both the early and advanced-stages [6, 7].However, patients without complete response make up approximately $80 \%$ of TNBC [8], and there is a rapid recurrence and metastasis [9]. Therefore, it is essential to excavate novel biomarkers to guide the treatment and improve the clinical management of TNBC. Histopathological examination of tumor biopsy specimen was considered as the gold standard for diagnosing TNBC. However, this approach has several limitations. First, owing to tumor heterogeneity, a biopsy specimen may not be representative of the entire tumor, let alone distant metastasis [10]. In addition, tissue processing, fixatives, and storage may diminish DNA quality and affect later mutation detection [11]. In the end, biopsy is a very invasive method and not always feasible in clinical practice. Currently, TNBC patients have no unanimous biomarkers that would aid in diagnosing, treating, and determining the prognosis of TNBC [12]. Novel predictive biomarkers commited to patients 
suffering from TNBC should be urgently needed. Plasma circulating tumor DNA (ctDNA), including genetic information about not only the primary tumor but also metastatic disease [13-15], could be used as a non-invasive practice and might be a good surrogate to the current solid biopsy and help in TNBC management.

In contrast to tissue biopsy, "liquid biopsy" is performed on cancer-derived material captured in a blood sample. Because it acts as a repository of genetic material from throughout the body, circulating blood may provide material for more comprehensive mutation profiling. Furthermore, "liquid biopsy" provides a non-invasive means of obtaining timely, comprehensive information and could provide the foundation for real-time tumor monitoring. Finally, research shows that tissue and liquid biopsies have excellent concordance in terms of gene mutation status by using BEAMing that could be theoretically conducted in lieu of a biopsy [16]. Recently, Garcia-Murillas et al. [17] and Olsson et al. [18] showed that using ctDNA-based liquid biopsy for real-time disease monitoring may improve the clinical management of breast cancer. These studies have indicated that sufficient sensitivity and specificity were eventually confirmed in terms of predicting an early recurrence with ctDNA, which might make it a monitoring tool for early tumor detection, therapy modification, and prognostic evaluation. Several small clinical trials have investigated the potential use of ctDNA as a biomarker for early breast cancer screening, monitoring treatment response, and determining prognosis $[17,19-21]$. In this review, we will highlight the latest research of ctDNA as a "liquid biopsy" in TNBC, and what role ctDNA plays in improving the clinical management of TNBC.

\section{Detection of ctDNA}

Malignant tumors can release significant amounts of DNA, including fragments as large as 180-200bp, into the bloodstream via cell deathassociated mechanisms such as necrosis and apoptosis [22-25]. Researchers can derive mutation information from such ctDNA by measuring its concentration and determining its nucleotide sequence [26]. Although highly sensitive and specific methods have been developed to detect ctDNA, ultrasensitive technologies that can detect the minimum amount of ctDNA in a sea of normal ctDNA are urgently needed for early cancer detection because of its very low levels of ctDNA. Such detection requires technology standardization coupled with accurate criteria to determine sample adequacy $[27,28]$.

\section{Screening}

Previous studies have indicated that an increase in ctDNA reflects an increase in tumor burden [29] and patients with early-stage breast cancer, therefore, may have only low concentrations of ctDNA. Owing to little information about genomic mutations it provided, ctDNA is rarely used as a biomarker for early diagnosis. In terms of early-stage TNBC, biomarkers as a means of screening disease was mainly investigated at the tissue DNA in TNBC [30, 31].For example, Elena et al. analysis showed that the acquisition of TP53 mutations may lead to increased genetic instability and increase the likelihood of an microglandular adenosis (MGA) and/or atypical MGA (AMGA) to progress to TNBC [31]. In this context, they collected two pure MGAs and eight cases of MGA and/or AMGA associated with in situ or invasive TNBC and subjected to massively parallel sequencing. They found that TNBCs are often clonally associated with synchronously diagnosed ipsilateral MGA and/or AMGA, providing circumstantial evidence to indicate that MGA may constitutes a non-obligate precursor of TNBC.

Although limited data are available for TNBC specifically, the validity of using ctDNA for the early diagnosis of breast cancer in general has already been studied. For example, Phallen et al. used massively parallel sequencing to detect ctDNA based on targeted error correction sequencing [21]. They assessed plasma samples from 200 patients with stage I or II colorectal, breast, lung, or ovarian cancer for somatic mutations. Thereinto, the authors found high concordance between ctDNA and breast tumor samples in terms of mutational status, which suggests that ctDNA analysis may provide a noninvasive means for early breast cancer screening. Similarly, a recent study demonstrated the possible implementtation of plasma tumor DNA detection in patients with early-stage breast cancer [32]. The authors assessed the feasibility of detecting PIK3CA mutations in the plasma of 29 patients before surgery and found 93\% concordance between confirmed mutations in tumor tissues and mutations in pre-surgery plasma samples. The high level of sensitivity $(93.3 \%)$ and specificity $(100 \%)$ of the ddPCR in this study provides a proof of concept and confirms that ctDNA analysis is feasible for patients with early-stage breast cancer. Another study showed that quantitative analysis of the methylation pattern of plasma cell-free DNA (cfDNA) in breast cancer patients might be a valuable non-invasive tool for early detection [33]. Due to limited data, as a novel biomarker for the early detection of breast cancer, ctDNA needs further development and validation. 


\section{Monitoring treatment response}

Conventional chemotherapy with anthracyclines, taxanes, and/or platinum agents is the current standard of care for TNBC patients [34]. Unfortunately, because of disease heterogeneity, not all TNBC patients have a good response to chemotherapy. Currently, whether treatment should be personalized for patients with different TNBC subtypes remains unclear $[35,36]$. Moreover, there are no guidelines or regulatory approvals for the use of ctDNA-based liquid biopsies to monitor treatment response in TNBC patients. Therefore, ctDNA concentration and sequencing analysis requires extensive study before it can be used with confidence to make treatment decisions for TNBC patients.

\section{Early stage}

CtDNA can be detected in the plasma and serum of patients with advanced cancer [37] and thus may be a noninvasive source to characterize the somatic genetic features of their tumors [38-40]. Data about whether ctDNA analyses would be applicable to early-stage cancer are limited in part because the low tumor burden of early-stage disease makes the detection of ctDNA challenging [32, 41], as very low levels of plasma ctDNA are usually not discoverable $[42,43]$. Nevertheless, in the setting of primary breast cancer, the clinical utility of ctDNA-based liquid biopsy remains unclear, as the percutaneous biopsy of breast tumors is preferred.

However, using ctDNA for the dynamic detection of treatment response can establish the rationale for using ctDNA-based liquid biopsy to detect early metastasis, inform timely therapy modification, and avoid overtreatment in patients with early-stage breast cancer. Kim et al. [44] using gene expression profiling and ER protein assays, found that the low-level expression of ESR1 is correlated with tamoxifen resistance in ER-positive primary breast cancer and is associated with treatment outcome. In another study designed to monitor therapy response in patients with early-stage breast cancer. Olsson et al. [18] demonstrated that tumor-specific chromosomal rearrangements in ctDNA could be detected on average 11 months before the clinical observation of metastases.

One study showed a remarkable correlation of methylated ctDNA with the primary breast tumor's response to neoadjuvant chemotherapy [45]. The authors concluded that methylated ctDNA is more sensitive than carcinoembryonic antigen or cancer antigen CA15-3 as a marker of sensitivity to neoadjuvant chemotherapy. In clinical practice, TNBC typically display larger tumor size and higher proliferation at the time of diagnosis, and are often treated by neoadjuvant chemotherapy (NCT) before surgery. However, only $20-30 \%$ of patients with TNBC achieve pathological complete response (pCR) following NCT, meanwhile the response to NCT was highly divergent $[46,47]$. Previous evidence supporting the association between O6-Methylguanine-DNA methyltransferase (MGMT) expression status and achievement of pCR following NCT in basal-like breast cancers (BLBCs) comes from Katsuya et al., who showed that attenuated expression of MGMT is predictive of a pCR [48]. In a study of 36 TNBC patients, Riva.F et al. using ddPCR, detected TP53 mutations in all patients' plasma before NCT, after 1 cycle of neoadjuvant chemotherapy, before surgery, and after surgery to assess tumor response to NCT [49]. Notably, 27 of 36 patients (75\%) had TP53 mutations at baseline. During the treatment, all patients' ctDNA levels declined, and no patients had detectable ctDNA after surgery. One patient with rising ctDNA levels experienced tumor progression during NCT. In this study, Riva.F et al. demonstrated that the detection and quantification of ctDNA is a very promising tool for assessing response to NCT. Another study drew similar conclusions. Chen, Y. H. et al. [50] followed 38 early-stage triple-negative breast cancers and utilized next-generation in matched tumor, blood and plasma to detect recurrence and metastasis as early as possible. Of those 33 patients who had primary tumor mutation, 4 had ctDNA mutations. For those patients where ctDNA mutation was detected, recurrence was rapid. However, in next few studies, the results have been disappointing. Of the 84 TNBC patients treated with alkylating agents in the NCT setting, 58.3\% have MGMT methylation in addition to the status of unmethylated $(27.4 \%)$ or indeterminate $(14.3 \%)$. Confirming these data, Caterina and colleagues found that although high methylation levels in FFPE samples were association with clinical response to therapy, no association between MGMT methylation status and $\mathrm{pCR}$ was found [51]. The association between gene mutations and NCT response was also seen in other primary tumor studies. Using next generation sequencing, 1,977 genes were analyzed from 56 pre-treatment TNBC biopsies before treatment. Lips et al. [52] found that TP53 mutations are the most frequent clonal event (55\%), followed by TTN mutations (14\%) and PIK3CA mutations (9\%). However, few mutations were common among the samples, and they were unable to find any recurrent mutations associated with NCT response. Further, they observed no difference in the mutation rates between responders and non-responders. A similar finding was reported from a subsequent analysis 
between TP53 mutations and the response to NCT in biopsy tissue from TNBC [53]. In this study, Silvia et al. [53] indicated that TP53 mutations did not predict the NCT response in TNBC patients. Furthermore, analysis of the predictive of PIK3CA mutation on TNBC agents was performed in a biomarker analysis of 81 patients with residual disease after NCT, with finding of PIK3CA mutation not associated with $\mathrm{pCR}$ [54].

\section{Advanced stage}

Measuring treatment response in patients with metastatic breast cancer is usually done by serial clinical evaluation, the assessment of the serum CA15-3 levels, and serial radiographic imaging. However, serial radiographic imaging is expensive, often inconclusive, and may not detect changes in tumor burden. CA15-3 assessment has low sensitivity, and changes in CA15-3 levels do not necessarily reflect tumor response or progression [55]. Tampellini et al. [56] revealed that CA15-3 alone is not usable as a biomarker for monitoring therapy response in patients with advanced breast cancer.

Because no marker for monitoring therapy response in patients with metastatic breast cancer has yet reached wide clinical use, researchers hope to use ctDNA mutations as biomarkers for dynamically detecting treatment response. Dawson et al. [29] suggested that ctDNA analysis has great potential for the real-time monitoring of tumor burden and may be a better measure of treatment effectiveness in patients with metastatic disease. Several recent studies have investigated the ctDNA mutations associated with targeted therapy response in patients with HER2-positive breast cancer and endocrine therapy response in patients with ER-positive metastatic breast cancer [57-59]. For example, the ESR1 mutation has attracted special attention as a mechanism of endocrine therapy resistance in metastatic breast cancer. In a study evaluating the use of NGS- and ddPCR-based techniques to detect ESR1 mutations in the cfDNA of 48 patients with ER-positive breast cancer, Guttery et al. [57] demonstrated that ESR1 mutations mainly arise following the treatment of metastatic disease and can predict resistance to aromatase inhibitor-based therapy. Similarly, the detection of cfDNA nucleotide alterations to assess response to anti-HER2-targeted therapies has been investigated. In one study using NGS, 46 genes were detected from an assessment of 486 single-nucleotide variants [59]. Notably, only 7 genes considered relevant to targeted therapy resistance were detected in the treatment-resistant group. In addition, two patients in whom HER2 S855I mutations were detected derived sustained benefit from anti-HER2 therapy.

Overall, patients with TNBC do not benefit from endocrine or targeted therapy. Furthermore, TNBC, especially metastatic TNBC, treated with chemotherapy can progress without warning. Thus, a new sensitive hematological tumor biomarker of chemotherapy response in patients with metastatic TNBC is urgently needed. CtDNA analysis can represent an alternative to metastatic biopsies in molecular screening programs [60, 61]. Currently, ctDNA may be used to identify molecular alterations of immunotherapy effect in patients of metastatic TNBC. Results have reported that ctDNA detected the response of anti-PD1 immunotherapy of patients with nonsmall cell lung cancer, metastatic melanoma and other malignancies [62-66]. The programmed death receptor 1 (PD-1) is an inhibitory immune checkpoint receptor that can limit autoimmunity, regulate the activity of effector $\mathrm{T}$ cells in the periphery in response to an inflammatory stimulus $[67,68]$. Research shows that programmed death 1 ligand 1 (PD-L1) is expressed in approximately $20 \%$ of TNBC, suggesting anti-PD-L1/anti-PD1 therapy may play a potential role in this patient population [69]. Furthermore, the above studies have shown a correlation between serial analysis of ctDNA and tumor response to anti-PD-1 therapy. It therefore appears that ctDNA may be a useful test for predicting response to immunotherapy in metastatic TNBC. However, no studies have ever published in this field.

\section{Assessing prognosis}

Many studies have investigated the prognostic value of ctDNA in multiple cancers, including medullary thyroid carcinoma [70], pancreatic cancer [71], lung adenocarcinoma [72], gastric cancer [73], and hepatocellular carcinoma [74]. Although detectable levels of unmutated ctDNA in breast cancer patients have been related to prognosis, studies investigating the prognostic value of mutated cfDNA levels in breast cancer patients have provided mixed results. Visvanathan et al. found that a high cumulative methylation index in cfDNA was associated with shorter median PFS and overall survival (OS) of breast cancer patients. They also found that high cumulative methylation index levels at week 4 were related to worse PFS [75]. In a meta-analysis involving 3,915 breast cancer patients, Sheng et al. [76] found that ESR1 and PITX2 promoter methylation were linked to worse OS. In contrast, Huang et al. [77] found no association between plasma ctDNA levels and clinicopathological parameters. Another study also yielded ambiguous results. Iqbal et al. [19] found no significant difference in the OS durations of breast cancer patients based on 
their baseline levels of ALU115, ALU247, and DNA integrity.

With the rapid development of NGS technologies, the obstacle of identifying highly diluted ctDNA among normal germline cfDNA has been circumvented [78]. Recent evidence has shown that ctDNA sequencing may have prognostic value in predicting residual disease after neoadjuvant chemotherapy with high specificity [50]. Several novel biomarkers of TNBC, some of which remain controversial for assessing prognosis, must be elucidated. In early-stage TNBC, a previous subgroup analysis of a retrospective study in patients with TNBC who received adjuvant chemotherapy after surgery, low mRNA expression in patients with TP53 missense mutation was associated with poor prognosis [79]. In this study, Ji-Yeon Kim et al. concluded that TP53 mutation and its expression were a potential prognostic marker of TNBC. An in-depth study of ctDNA mutation was performed by Takeshita et al. [80], who used ddPCR of cfDNA to determine the PIK3CA mutation status of 49 patients with early-stage TNBC. Of these 49 patients, 12 (24\%) had PIK3CA mutations and were followed for a median time of 54.4 months. Takeshita et al. confirmed that PIK3CA mutations are correlated with relapse-free survival and breast cancer-specific survival and demonstrated that PIK3CA mutations in TNBC are related to PI3K pathway-dependent androgen receptor phosphorylation, which is considered to be an independent prognostic factor for TNBC. In metastatic TNBC, the prognostic value of ctDNA was contradictory. Madic et al. [81] found that the baseline ctDNA levels of patients with metastatic TNBC were not predictive of radiological tumor response and were not correlated with time to progression or OS duration. They noted that because the prognostic value of ctDNA is still under evaluation, further exploration in TNBC is urgently needed. However, recent findings of a retrospective cohort study assessing the prognostic effect of ctDNA to characterize somatic copy number alterations (SCNAs) and quantify tumor fraction (TFx) in metastatic TNBC.Using low-coverage (0.13) whole-genome sequencing (WGS), Stover et al. [82] profile SCNAs and TFx, they found that certain SCNAs including chromosomal gains in drivers $\mathrm{NOTCH} 2, \mathrm{AKT} 2$, and AKT3 were more frequent in metastatic TNBC versus primary TNBC. Moreover, gain or amplification at 18q11 and 19p13 identified a subset of TNBC with poor prognosis in the metastatic setting. In addition to evaluate SCNA differences in primary versus metastatic TNBCs, they also identified that patients with $\mathrm{TF} x \geq 10 \%$ had significantly inferior survival and remained significant independent of clinicopathologic factors. In view of the above results, although it is essential to continue a further study including larger numbers of specimens, we have abundant reasons to believe that ctDNA mutations can serve as a prognostic information for TNBC.

\section{Conclusion}

A highly sensitive ctDNA-based system is urgently needed in further studies for early detection and management of TNBC patients. Patients with TNBC have increased risk of relapse and inferior outcomes. Despite the aggressive biology and poor prognosis associated with TNBC, currently, there are no acknowledged ctDNA-based targeted treatment regimens available for patients with this disease subtype. This lack of targeted therapy and detrimental biology highlight the urgent need for a novel biomarker to facilitate a more tailored treatment approach to improve outcomes for patients with TNBC. Several studies have characterized genes and pathways in TNBC that may be targeted therapeutically [83-87], and these drug targets, coupled with improved ctDNA detection at all stages of disease, will improve the management of TNBC patients considerably.

Currently, despite the great progress made in exploiting ctDNA for determining prognosis and assessing NCT response in patients of TNBC, ctDNA is rarely used for early disease screening and the detection of the response to treatment of advanced stage. The hope that the early detection of TNBC, when the disease is most amenable to cure, can afford opportunities for treatment while sparing patients from overtreatment has not yet been realized. Several studies with small samples have already demonstrated the use of ctDNA in the management of TNBC, but the extent to which the detection of ctDNA influences treatment decisions and affects survival outcomes remains unclear. Moreover, the amount of ctDNA can greatly vary as time goes on, demanding abundant molecular detection technologies able to detect them even when present at low levels. In the meantime, there is a great heterogeneity among the ctDNA data in different studies, technical platforms and patient populations. A standardization of cfDNA analyses in terms of sample collection, processing, and molecular techniques is needed. Finally, there is currently no industry standard or widespread clinical acceptance for the use of ctDNA, nor a uniformly agreed upon platform in TNBC. In the TNBC research setting, there are many barriers to universal clinical implementation of detection in ctDNA, including the need for plasma samples, cost of testing, lack of submitting an expense account, and poor understanding of the clinical implications associated 
with tumor mutations. In order to advance our knowledge of this complicated and everchanging field, we desperately need large, international, multi-institutional cooperative trials that will allow us to enroll patients with less common germline mutations to accomplish the process of TNBC diagnosis to treatment to evaluate prognosis.

\section{Abbreviations}

AMGA: atypical microglandular adenosis; BLBCs: basal-like breast cancers; cfDNA: cell-free DNA; ctDNA: circulating tumor DNA; ER: estrogen receptor; HER2: human epidermal growth factor receptor 2; MGA: microglandular adenosis; MGMT: O6-Methylguanine-DNA methyltransferase; NCT: neoadjuvant chemotherapy; OS: overall survival; pCR: pathological complete response; PD-1: programmed death receptor 1; PD-L1: programmed death 1 ligand 1; PFS: progression-free survival; PR: progesterone receptor; SCNAs: somatic copy number alterations; TFx: tumor fraction; TNBC: triple-negative breast cancer; WGS: whole-genome sequencing.

\section{Acknowledgements}

We would like to acknowledge the funding support of Natural Science Foundation of Shandong Province (Grant No. ZR2016HM41 and ZR2015HZ 004), National Natural Science Foundation of China (Grant No. 81472812) and Key Research and Development Program of Shandong Province (Grant No. 2018GSF118089).

\section{Competing Interests}

The authors have declared that no competing interest exists.

\section{References}

1. Blows FM, Driver KE, Schmidt MK, Broeks A, van Leeuwen FE, Wesseling J, et al. Subtyping of breast cancer by immunohistochemistry to investigate a relationship between subtype and short and long term survival: a collaborative analysis of data for 10,159 cases from 12 studies. PLoS medicine. 2010; 7: e1000279.

2. Lehmann BD, Bauer JA, Chen X, Sanders ME, Chakravarthy AB, Shyr $Y$, et al Identification of human triple-negative breast cancer subtypes and preclinical models for selection of targeted therapies. The Journal of clinical investigation. 2011; 121: 2750-67.

3. Metzger-Filho O, Tutt A, de Azambuja E, Saini KS, Viale G, Loi S, et al. Dissecting the heterogeneity of triple-negative breast cancer. Journal of clinical oncology : official journal of the American Society of Clinical Oncology. 2012; 30: 1879-87.

4. Schmadeka R, Harmon BE, Singh M. Triple-negative breast carcinoma: current and emerging concepts. American journal of clinical pathology. 2014; 141: $462-77$.

5. Zaharia M, Gomez H. [Triple negative breast cancer: a difficult disease to diagnose and treat]. Revista peruana de medicina experimental y salud publica. 2013; 30: 649-56

6. Peto R, Davies C, Godwin J, Gray R, Pan HC, Clarke M, et al. Comparisons between different polychemotherapy regimens for early breast cancer: meta-analyses of long-term outcome among 100,000 women in 123 randomised trials. Lancet (London, England). 2012; 379: 432-44.

7. Cortazar P, Zhang L, Untch M, Mehta K, Costantino JP, Wolmark N, et al. Pathological complete response and long-term clinical benefit in breast cancer: the CTNeoBC pooled analysis. Lancet (London, England). 2014; 384: 164-72.
8. Jhan JR, Andrechek ER. Triple-negative breast cancer and the potential for targeted therapy. Pharmacogenomics. 2017; 18: 1595-609.

9. Haffty BG, Yang Q, Reiss M, Kearney T, Higgins SA, Weidhaas J, et al. Locoregional relapse and distant metastasis in conservatively managed triple negative early-stage breast cancer. Journal of clinical oncology : official journal of the American Society of Clinical Oncology. 2006; 24: 5652-7.

10. Chan KC, Jiang P, Zheng YW, Liao GJ, Sun H, Wong J, et al. Cancer genome scanning in plasma: detection of tumor-associated copy number aberrations, single-nucleotide variants, and tumoral heterogeneity by massively parallel sequencing. Clinical chemistry. 2013; 59: 211-24.

11. Srinivasan M, Sedmak D, Jewell S. Effect of fixatives and tissue processing on the content and integrity of nucleic acids. The American journal of pathology. 2002; 161: 1961-71.

12. Andreopoulou E, Kelly CM, McDaid HM. Therapeutic Advances and New Directions for Triple-Negative Breast Cancer. Breast care (Basel, Switzerland). 2017; 12: 21-8.

13. Diaz LA, Jr., Bardelli A. Liquid biopsies: genotyping circulating tumor DNA. Journal of clinical oncology : official journal of the American Society of Clinical Oncology. 2014; 32: 579-86.

14. Zardavas D, Irrthum A, Swanton C, Piccart M. Clinical management of breast cancer heterogeneity. Nature reviews Clinical oncology. 2015; 12: 381-94.

15. Rivenbark AG, O'Connor SM, Coleman WB. Molecular and cellular heterogeneity in breast cancer: challenges for personalized medicine. The American journal of pathology. 2013; 183: 1113-24.

16. Higgins MJ, Jelovac D, Barnathan E, Blair B, Slater S, Powers P, et al. Detection of tumor PIK3CA status in metastatic breast cancer using peripheral blood. Clinical cancer research : an official journal of the American Association for Cancer Research. 2012; 18: 3462-9.

17. Garcia-Murillas I, Schiavon G, Weigelt B, Ng C, Hrebien S, Cutts RJ, et al. Mutation tracking in circulating tumor DNA predicts relapse in early breast cancer. Science translational medicine. 2015; 7: 302ra133.

18. Olsson E, Winter C, George A, Chen Y, Howlin J, Tang MH, et al. Serial monitoring of circulating tumor DNA in patients with primary breast cancer for detection of occult metastatic disease. EMBO molecular medicine. 2015; 7: 1034-47.

19. Iqbal S, Vishnubhatla S, Raina V, Sharma S, Gogia A, Deo SS, et al. Circulating cell-free DNA and its integrity as a prognostic marker for breast cancer. SpringerPlus. 2015; 4: 265.

20. Zhou B, Xin L, Xu L, Liu YH, Zhang MM, Jing RL, et al. [Exploratory study of circulating tumor DNA detection in early breast cancer: an analysis of 75 next-generation sequencing results]. Zhonghua wai ke za zhi [Chinese journal of surgery]. 2017; 55: 847-52.

21. Phallen J, Sausen M, Adleff V, Leal A. Direct detection of early-stage cancers using circulating tumor DNA. 2017; 9 .

22. Crowley E, Di Nicolantonio F, Loupakis F, Bardelli A. Liquid biopsy: monitoring cancer-genetics in the blood. Nature reviews Clinical oncology. 2013; 10: 472-84.

23. Gormally E, Hainaut P, Caboux E, Airoldi L, Autrup H, Malaveille C, et al. Amount of DNA in plasma and cancer risk: a prospective study. International journal of cancer. 2004; 111: 746-9.

24. Jahr S, Hentze H, Englisch S, Hardt D, Fackelmayer FO, Hesch RD, et al. DNA fragments in the blood plasma of cancer patients: quantitations and evidence for their origin from apoptotic and necrotic cells. Cancer research. 2001; 61: 1659-65.

25. Mouliere F, Robert B, Arnau Peyrotte E, Del Rio M, Ychou M, Molina F, et al. High fragmentation characterizes tumour-derived circulating DNA. PloS one. 2011; 6: e23418.

26. Frattini M, Gallino G, Signoroni S, Balestra D, Lusa L, Battaglia L, et al. Quantitative and qualitative characterization of plasma DNA identifies primary and recurrent colorectal cancer. Cancer letters. 2008; 263: 170-81.

27. Schwarzenbach $\mathrm{H}$, Hoon DS, Pantel K. Cell-free nucleic acids as biomarkers in cancer patients. Nature reviews Cancer. 2011; 11: 426-37.

28. De Mattos-Arruda L, Cortes J, Santarpia L, Vivancos A, Tabernero J, Reis-Filho JS, et al. Circulating tumour cells and cell-free DNA as tools for managing breast cancer. Nature reviews Clinical oncology. 2013; 10: 377-89.

29. Dawson SJ, Tsui DW, Murtaza M, Biggs H, Rueda OM, Chin SF, et al. Analysis of circulating tumor DNA to monitor metastatic breast cancer. The New England journal of medicine. 2013; 368: 1199-209.

30. Geyer FC, Lacroix-Triki M, Colombo PE, Patani N, Gauthier A, Natrajan R, et al. Molecular evidence in support of the neoplastic and precursor nature of microglandular adenosis. Histopathology. 2012; 60: E115-30.

31. Guerini-Rocco E, Piscuoglio S, Ng CK, Geyer FC, De Filippo MR, Eberle CA, et al. Microglandular adenosis associated with triple-negative breast cancer is a neoplastic lesion of triple-negative phenotype harbouring TP53 somatic mutations. The Journal of pathology. 2016; 238: 677-88.

32. Beaver JA, Jelovac D, Balukrishna S, Cochran R, Croessmann S, Zabransky DJ, et al. Detection of cancer DNA in plasma of patients with early-stage breast cancer. Clinical cancer research : an official journal of the American Association for Cancer Research. 2014; 20: 2643-50.

33. Li Z, Guo X, Tang L, Peng L, Chen M, Luo X, et al. Methylation analysis of plasma cell-free DNA for breast cancer early detection using bisulfite next-generation sequencing. Tumour biology : the journal of the International Society for Oncodevelopmental Biology and Medicine. 2016; 37: 13111-9. 
34. Shah SP, Roth A, Goya R, Oloumi A, Ha G, Zhao Y, et al. The clonal and mutational evolution spectrum of primary triple-negative breast cancers. Nature. 2012; 486: 395-9.

35. Berrada N, Delaloge S, Andre F. Treatment of triple-negative metastatic breast cancer: toward individualized targeted treatments or chemosensitization? Annals of oncology : official journal of the European Society for Medical Oncology. 2010; 21 Suppl 7: vii30-5.

36. Fornier M, Fumoleau P. The paradox of triple negative breast cancer: novel approaches to treatment. The breast journal. 2012; 18: 41-51.

37. Leon SA, Shapiro B, Sklaroff DM, Yaros MJ. Free DNA in the serum of cancer patients and the effect of therapy. Cancer research. 1977; 37: 646-50.

38. Gevensleben H, Garcia-Murillas I, Graeser MK, Schiavon G, Osin P, Parton M, et al. Noninvasive detection of HER2 amplification with plasma DNA digital PCR. Clinical cancer research : an official journal of the American Association for Cancer Research. 2013; 19: 3276-84

39. Forshew T, Murtaza M, Parkinson C, Gale D, Tsui DW, Kaper F, et al. Noninvasive identification and monitoring of cancer mutations by targeted deep sequencing of plasma DNA. Science translational medicine. 2012; 4: 136ra68

40. Murtaza M, Dawson SJ, Tsui DW, Gale D, Forshew T, Piskorz AM, et al. Non-invasive analysis of acquired resistance to cancer therapy by sequencing of plasma DNA. Nature. 2013; 497: 108-12.

41. Bettegowda C, Sausen M, Leary RJ, Kinde I, Wang Y, Agrawal N, et al. Detection of circulating tumor DNA in early- and late-stage human malignancies. Science translational medicine. 2014; 6: 224ra24.

42. Diehl F, Schmidt K, Choti MA, Romans K, Goodman S, Li M, et al. Circulating mutant DNA to assess tumor dynamics. Nature medicine. 2008; 14: 985-90.

43. Reinert $\mathrm{T}$, Scholer LV, Thomsen R, Tobiasen $\mathrm{H}$, Vang S, Nordentoft $\mathrm{I}$, et al Analysis of circulating tumour DNA to monitor disease burden following colorectal cancer surgery. Gut. 2016; 65: 625-34.

44. Kim C, Tang G, Pogue-Geile KL, Costantino JP, Baehner FL, Baker J, et al. Estrogen receptor (ESR1) mRNA expression and benefit from tamoxifen in the treatment and prevention of estrogen receptor-positive breast cancer. Journal of clinical oncology : official journal of the American Society of Clinical Oncology. 2011; 29: 4160-7.

45. Takahashi H, Kagara N, Tanei T, Naoi Y, Shimoda M, Shimomura A, et al. Correlation of Methylated Circulating Tumor DNA With Response to Neoadjuvant Chemotherapy in Breast Cancer Patients. Clinical breast cancer. 2017; 17: 61-9.e3

46. Liedtke C, Mazouni C, Hess KR, Andre F, Tordai A, Mejia JA, et al. Response to neoadjuvant therapy and long-term survival in patients with triple-negative breast cancer. Journal of clinical oncology : official journal of the American Society of Clinical Oncology. 2008; 26: 1275-81.

47. Dawood S, Broglio K, Kau SW, Green MC, Giordano SH, Meric-Bernstam F, et al. Triple receptor-negative breast cancer: the effect of race on response to primary systemic treatment and survival outcomes. Journal of clinical oncology : official journal of the American Society of Clinical Oncology. 2009; 27: 220-6

48. Nakai K, Mitomi H, Alkam Y, Arakawa A, Yao T, Tokuda E, et al. Predictive value of MGMT, hMLH1, hMSH2 and BRCA1 protein expression for pathological complete response to neoadjuvant chemotherapy in basal-like breast cancer patients. Cancer chemotherapy and pharmacology. 2012; 69: 923-30

49. Riva F, Bidard FC, Houy A, Saliou A, Madic J, Rampanou A, et al. Patient-Specific Circulating Tumor DNA Detection during Neoadjuvant Chemotherapy in Triple-Negative Breast Cancer. Clinical chemistry. 2017; 63: 691-9.

50. Chen YH, Hancock BA, Solzak JP, Brinza D, Scafe C, Miller KD, et al. Next-generation sequencing of circulating tumor DNA to predict recurrence in triple-negative breast cancer patients with residual disease after neoadjuvant chemotherapy. NPJ breast cancer. 2017; 3: 24

51. Fumagalli C, Della Pasqua S, Bagnardi V, Cardillo A, Sporchia A, Colleoni M, et al. Prevalence and clinicopathologic correlates of $\mathrm{O}(6)$-methylguanine-DNA methyltransferase methylation status in patients with triple-negative breast cancer treated preoperatively by alkylating drugs. Clinical breast cancer. 2014; 14: $285-90$

52. Lips EH, Michaut M, Hoogstraat M, Mulder L, Besselink NJ, Koudijs MJ, et al. Next generation sequencing of triple negative breast cancer to find predictors for chemotherapy response. Breast cancer research : BCR. 2015; 17: 134

53. Darb-Esfahani S, Denkert C, Stenzinger A, Salat C, Sinn B, Schem C, et al. Role of TP53 mutations in triple negative and HER2-positive breast cancer treated with neoadjuvant anthracycline/taxane-based chemotherapy. Oncotarget. 2016; 7: 67686-98

54. Castaneda CA, Lopez-Ilasaca M, Pinto JA, Chirinos-Arias M, Doimi F, Neciosup SP, et al. PIK3CA mutations in Peruvian patients with HER2-amplified and triple negative non-metastatic breast cancers. Hematology/oncology and stem cell therapy. 2014; 7: 142-8.

55. Duffy MJ, Evoy D, McDermott EW. CA 15-3: uses and limitation as a biomarker for breast cancer. Clinica chimica acta; international journal of clinical chemistry. 2010; 411: 1869-74.

56. Tampellini M, Berruti A, Bitossi R, Gorzegno G, Alabiso I, Bottini A, et al. Prognostic significance of changes in CA 15-3 serum levels during chemotherapy in metastatic breast cancer patients. Breast cancer research and treatment. 2006; 98: 241-8.
57. Guttery DS, Page K, Hills A, Woodley L, Marchese SD, Rghebi B, et al. Noninvasive detection of activating estrogen receptor 1 (ESR1) mutations in estrogen receptor-positive metastatic breast cancer. Clinical chemistry. 2015; 61: 974-82.

58. Schiavon G, Hrebien S, Garcia-Murillas I, Cutts RJ, Pearson A, Tarazona N, et al. Analysis of ESR1 mutation in circulating tumor DNA demonstrates evolution during therapy for metastatic breast cancer. Science translational medicine. 2015; 7: 313ra182.

59. Ye Q, Qi F, Bian L, Zhang SH, Wang T, Jiang ZF. Circulating-free DNA Mutation Associated with Response of Targeted Therapy in Human Epidermal Growth Factor Receptor 2-positive Metastatic Breast Cancer. Chinese medical journal. 2017; 130: 522-9.

60. Rothe F, Laes JF, Lambrechts D, Smeets D, Vincent D, Maetens M, et al. Plasma circulating tumor DNA as an alternative to metastatic biopsies for mutational analysis in breast cancer. Annals of oncology : official journal of the European Society for Medical Oncology. 2014; 25: 1959-65.

61. Mattos-Arruda L, Weigelt B, Cortes J, Won $\mathrm{HH}, \mathrm{Ng}$ CKY, Nuciforo $\mathrm{P}$, et al. Capturing intra-tumor genetic heterogeneity by de novo mutation profiling of circulating cell-free tumor DNA: a proof-of-principle. Annals of oncology : official journal of the European Society for Medical Oncology. 2018.

62. Cabel L, Riva F, Servois V, Livartowski A, Daniel C, Rampanou A, et al Circulating tumor DNA changes for early monitoring of anti-PD1 immunotherapy: a proof-of-concept study. Annals of oncology : official journal of the European Society for Medical Oncology. 2017; 28: 1996-2001.

63. Iijima Y, Hirotsu Y, Amemiya K, Ooka Y, Mochizuki H, Oyama T, et al. Very early response of circulating tumour-derived DNA in plasma predicts efficacy of nivolumab treatment in patients with non-small cell lung cancer. European journal of cancer (Oxford, England : 1990). 2017; 86: 349-57.

64. Guibert N, Mazieres J, Delaunay M, Casanova A, Farella M, Keller L, et al. Monitoring of KRAS-mutated ctDNA to discriminate pseudo-progression from true progression during anti-PD-1 treatment of lung adenocarcinoma. Oncotarget. 2017; 8: 38056-60.

65. Lee JH, Long GV, Boyd S, Lo S, Menzies AM, Tembe V, et al. Circulating tumour DNA predicts response to anti-PD1 antibodies in metastatic melanoma. Annals of oncology : official journal of the European Society for Medical Oncology. 2017; 28: 1130-6.

66. Khagi Y, Goodman AM, Daniels GA, Patel SP, Sacco AG, Randall JM, et al. Hypermutated Circulating Tumor DNA: Correlation with Response to Checkpoint Inhibitor-Based Immunotherapy. Clinical cancer research : an official journal of the American Association for Cancer Research. 2017; 23: 5729-36.

67. Pardoll DM. The blockade of immune checkpoints in cancer immunotherapy. Nature reviews Cancer. 2012; 12: 252-64

68. Keir ME, Butte MJ, Freeman GJ, Sharpe AH. PD-1 and its ligands in tolerance and immunity. Annual review of immunology. 2008; 26: 677-704.

69. Mittendorf EA, Philips AV, Meric-Bernstam F, Qiao N, Wu Y, Harrington S, et al. PD-L1 expression in triple-negative breast cancer. Cancer immunology research. 2014; $2: 361-70$.

70. Cote GJ, Evers C, Hu MI, Grubbs EG, Williams MD, Hai T, et al. Prognostic Significance of Circulating RET M918T Mutated Tumor DNA in Patients With Advanced Medullary Thyroid Carcinoma. The Journal of clinical endocrinology and metabolism. 2017; 102: 3591-9.

71. Pietrasz D, Pecuchet N, Garlan F, Didelot A, Dubreuil O, Doat S, et al. Plasma Circulating Tumor DNA in Pancreatic Cancer Patients Is a Prognostic Marker. Clinical cancer research : an official journal of the American Association for Cancer Research. 2017; 23: 116-23.

72. Wu YL, Sequist LV, Hu CP, Feng J, Lu S, Huang Y, et al. EGFR mutation detection in circulating cell-free DNA of lung adenocarcinoma patients: analysis of LUX-Lung 3 and 6. British journal of cancer. 2017; 116: 175-85.

73. Gao Y, Zhang K, Xi H, Cai A, Wu X, Cui J, et al. Diagnostic and prognostic value of circulating tumor DNA in gastric cancer: a meta-analysis. Oncotarget. 2017; 8: 6330-40.

74. Lu CY, Chen SY, Peng HL, Kan PY, Chang WC, Yen CJ. Cell-free methylation markers with diagnostic and prognostic potential in hepatocellular carcinoma. Oncotarget. 2017; 8: 6406-18.

75. Visvanathan K, Fackler MS, Zhang Z, Lopez-Bujanda ZA, Jeter SC, Sokoll LJ, et al. Monitoring of Serum DNA Methylation as an Early Independent Marker of Response and Survival in Metastatic Breast Cancer: TBCRC 005 Prospective Biomarker Study. Journal of clinical oncology : official journal of the American Society of Clinical Oncology. 2017; 35: 751-8.

76. Sheng X, Guo Y, Lu Y. Prognostic role of methylated GSTP1, p16, ESR1 and PITX2 in patients with breast cancer: A systematic meta-analysis under the guideline of PRISMA. Medicine. 2017; 96: e7476.

77. Huang ZH, Li LH, Hua D. Quantitative analysis of plasma circulating DNA at diagnosis and during follow-up of breast cancer patients. Cancer letters. 2006; 243. 64-70.

78. Parimi S, Ko JJ. Recent advances in circulating tumor cells and cell-free DNA in metastatic prostate cancer: a review. Expert review of anticancer therapy. 2017; 17: 939-49.

79. Kim JY, Park K, Jung HH, Lee E, Cho EY, Lee KH, et al. Association between Mutation and Expression of TP53 as a Potential Prognostic Marker of Triple-Negative Breast Cancer. Cancer research and treatment : official journal of Korean Cancer Association. 2016; 48: 1338-50. 
80. Takeshita T, Yamamoto Y, Yamamoto-Ibusuki M, Inao T, Sueta A, Fujiwara S, et al. Prognostic role of PIK3CA mutations of cell-free DNA in early-stage triple negative breast cancer. Cancer science. 2015; 106: 1582-9.

81. Madic J, Kiialainen A, Bidard FC, Birzele F, Ramey G, Leroy Q, et al. Circulating tumor DNA and circulating tumor cells in metastatic triple negative breast cancer patients. International journal of cancer. 2015; 136: 2158-65.

82. Stover DG, Parsons HA, Ha G, Freeman SS, Barry WT, Guo H, et al. Association of Cell-Free DNA Tumor Fraction and Somatic Copy Number Alterations With Survival in Metastatic Triple-Negative Breast Cancer. Journal of clinical oncology : official journal of the American Society of Clinical Oncology. 2018; 36: 543-53

83. Burstein MD, Tsimelzon A, Poage GM, Covington KR, Contreras A, Fuqua SA, et al. Comprehensive genomic analysis identifies novel subtypes and targets of triple-negative breast cancer. Clinical cancer research : an official journal of the American Association for Cancer Research. 2015; 21: 1688-98.

84. Hassan S, Esch A, Liby T, Gray JW, Heiser LM. Pathway-Enriched Gene Signature Associated with 53BP1 Response to PARP Inhibition in Triple-Negative Breast Cancer. Molecular cancer therapeutics. 2017; 16: 2892-901.

85. Afghahi A, Timms KM, Vinayak S, Jensen KC, Kurian AW, Carlson RW, et al. Tumor BRCA1 Reversion Mutation Arising during Neoadjuvant Platinum-Based Chemotherapy in Triple-Negative Breast Cancer Is Associated with Therapy Resistance. Clinical cancer research : an official journal of the American Association for Cancer Research. 2017; 23: 3365-70.

86. Sharpe R, Pearson A, Herrera-Abreu MT, Johnson D, Mackay A, Welti JC, et al. FGFR signaling promotes the growth of triple-negative and basal-like breast cancer cell lines both in vitro and in vivo. Clinical cancer research : an official journal of the American Association for Cancer Research. 2011; 17: 5275-86.

87. Frenel JS, Carreira S, Goodall J, Roda D, Perez-Lopez R, Tunariu N, et al. Serial Next-Generation Sequencing of Circulating Cell-Free DNA Evaluating Tumor Clone Response To Molecularly Targeted Drug Administration. Clinical cancer research : an official journal of the American Association for Cancer Research. 2015; 21: 4586-96. 